Vom 14.4. bis 16.4. führte uns die Fachexkursion an den Bodensee und nach Oberschwaben. Unser Organisations-Team hatte ein attraktives Programm zusammengestellt:

Aktuelle Geoinformationsprodukte und -dienste lernten wir beim Besuch von Airbus Defence \&t Space in Immenstaad durch unser Sektionsmitglied Hugh Sinclair und seinen Kollegen kennen. Eindrucksvoll war auch der Besuch der Satelliten-Montagehalle. Im Dornier Museum reisten wir durch 100 Jahre Luft- und Raumfahrt. Dietrich Diez führte uns durch Tettnang und im Museum Hopfengut $\mathrm{N}^{\circ} 20$ tauchten wir in die Welt des Hopfenanbaus und Bierbrauens ein. Der Heimweg führte uns zur Waldburg bei Ravensburg, Heimat der weltberühmten Waldseemüllerkarte. Bei einer Führung lernten wir die Burg kennen, den trigonometrischen Punkt der Landesvermessung und natürlich die kartographiehistorisch interessante Geschichte der Waldseemüller-Weltkarte. Den Abschluss bildete ein Besuch im Erwin Hymer Museum in Bad Waldsee mit der Ausstellung „Die ganze Welt des mobilen Reisens“. Präsentiert werden historische und aktuelle Wohnwagen und Reisemobile, aber auch Straßenund Traumrouten, sowie Reiseliteratur - auch die eine oder andere Karte war dabei.

Am 10.5.2016 führte uns der Weg zum Kampfmittelbeseitigungsdienst Baden-Württemberg ins Munitionslager in den Sindelfinger Wald. Siegfried Müller und seine Kollegen berichteten über die vielfältigen Aufgaben bei der Beseitigung von Kampfmitteln. Noch immer werden bei Bauarbeiten Blindgänger aus dem Zweiten Weltkrieg gefunden. Diese gilt es zu entschärfen, vernichten und das angefallene Material zu verwerten. Auch die Beschaffung und Auswertung von Luftbildaufnahmen der amerikanischen und britischen Luftwaffe, die nach den Angriffen gefertigt wurden spielt dabei eine wichtige Rolle.

Den Abschluss vor der Sommerpause bildete ein Besuch am 13.7. beim Württembergischen Kunstverein in Stuttgart. Hier wurden wir von Kurator Hans Christ durch die Ausstellung „Ein Loch im Meer" geführt. Die Ausstellung vermittelte uns ganz neue Perspektiven, welche unterschiedlichen Möglichkeiten es außer der Karte gibt um Raum darzustellen und zu erfahren. Mit Hilfe von Koordinaten, räumlicher Darstellung und einer ungewöhnlichen Straßenkartierung und weiteren Exponaten vermittelte Herr Christ uns einen für alle sehr interessanten Einblick in diese außergewöhnliche Ausstellung. Ganz besonders war er darüber erfreut, Kartographen als Fachleute für die Gestaltung des Raums als Gäste zu haben.

Aktuell haben wir 92 Mitglieder. Die Sektionsleitung ist unverändert: Wolfgang Schmid, Gernot Brück, Klaus-Peter Lawall, Reinhold Knödel.

Wolfgang Schmid, Stuttgart

\section{Langjährige DGfK-Mitgliedschaft}

November/Dezember 2016

Folgenden Mitgliedern dankt die Deutsche Gesellschaft für Kartographie für ihre langjährige Treue:

\section{Mitglied seit 60 Jahren:}

Dr. rer. nat. Heinrich Kötter, 79117 Freiburg

Dipl.-Ing. (FH) Reinhard K. Ryborsch, 63073 Offenbach am Main

Prof. Dr.-Ing. Heinz SchmidtFalkenberg, 83308 Trostberg

Mitglied seit 25 Jahren:

Dr.-Ing. Gerd Buziek,

83607 Holzkirchen

Dipl.-Ing. (FH) Christian Hild, 26340 Zetel

Dr. Uwe Schnall, 27612 Loxstedt

\section{Geburtstage}

\subsection{Prof. Dr.}

Jürgen Schweikart, 13437 Berlin, 60 Jahre

7.11. Univ.-Prof.

Dr. Jean-Claude Müller, 33334 Gütersloh, 75 Jahre

7.11. Dr. phil. Horst Schöttler, 67657 Kaiserslautern, 75 Jahre 9.11. Prof. Dr.-Ing.

Rudolf Schweißthal, 38300 Wolfenbüttel, 80 Jahre 10.11. Dieter Memminger, 71672 Marbach, 80 Jahre

11.11. Peter Rembold, 79249 Merzhausen, 91 Jahre 12.11. Egon Klemp, 13088 Berlin, 85 Jahre
14.11. Dipl.-Geogr. Wolfgang Meißner, 44287 Dortmund, 75 Jahre 21.11. Dipl.-Ing. (FH) Wolfgang Nitsche, 55130 Mainz-Weisenau, 65 Jahre

24.11. Dipl.-Ing. (FH)

Günter Huck, 65232 Taunusstein, 85 Jahre 25.11. Marianne KüstnerBrennemann, 20359 Hamburg, 70 Jahre

28.11. Prof. Dr.

Gerhard Pöhlmann, 14129 Berlin, 92 Jahre

9.12. Prof. Dr. Ulrich Freitag, 10587 Berlin, 85 Jahre

10.12. Dipl.-Ing. (FH)

Mario Rebetzky, 18057 Rostock, 60 Jahre

10.12. Dipl.-Ing. Jürgen Zedler 53340 Meckenheim, 75 Jahre 12.12. Dipl.-Ing. (FH)

Wolfgang Mehlitz,

61440 Oberursel am Taunus, 70 Jahre

12.12. Dr. Klaus Temlitz, 48161 Münster, 75 Jahre

14.12. Dr. rer.nat.

Heinrich Kötter,

79117 Freiburg, 94 Jahre

14.12. Dipl.-Ing.

Lorenz Lachart,

53639 Königswinter, 60 Jahre

18.12. Dipl.-Ing. (FH)

Stefan Dietrich, 44135 Dortmund, 65 Jahre

18.12. Dipl.-Ing.

Dieter Feuersenger, 53175 Bonn, 80 Jahre

30.12. Dipl.-Ing.

Helmut Zimmermann, 6179 Langenbogen, 80 Jahre

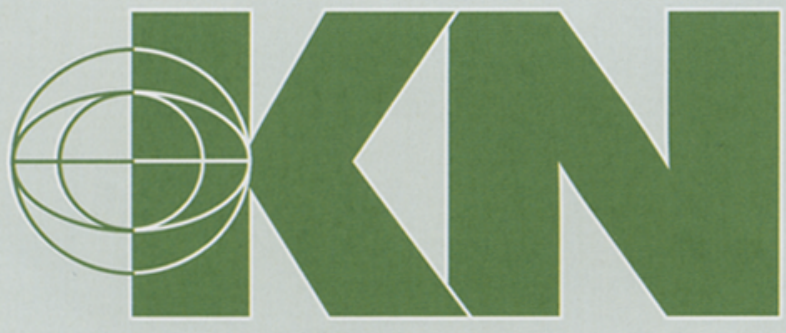

